\title{
Ovarian Pregnancy: a Rare Ectopic Pregnancy
}

\author{
Thapa $M,{ }^{1}$ Rawal $S,{ }^{1}$ Jha $R,{ }^{1}$ Singh $M^{1}$
}

'Department of Obstetrics and Gynaecology, TUTH, Kathmandu, Nepal.

\section{ABSTRACT}

Primary ovarian pregnancy is a rare entity, the incidence being 1 in 25,000 to 40,000 pregnancies. The diagnosis is difficult and still continues to challenge the clinicians. A case of ovarian pregnancy is reported here. The patient underwent laparotomy with right sided oophorectomy. Histopathological examination confirmed it to be an ovarian pregnancy.

Key Words: ectopic pregnancy, oophorectomy, ovarian pregnancy

\section{INTRODUCTION}

Ectopic pregnancy is an important health problem, accounting for about $10 \%$ of maternal mortality. ${ }^{1}$ Ovarian ectopic is a rare variant. Its incidence has been reported as 1 in 25,000 to 1 in 40,000 deliveries and accounts for 1 to $3 \%$ of all ectopic gestations. ${ }^{2}$

The first case was reported by St. Maurice in 1682 and many cases of ovarian ectopic pregnancy have been reported in the literature since then. Of late there has been an increase in the incidence of ovarian pregnancies due to better diagnostic modalities such as transvaginal ultrasonography and serum вhCG estimation. The increased incidence is because of wider use of intrauterine contraceptive device (IUCD), ovulatory drugs, and assisted reproductive techniques.
Correspondence:

Dr. Mira Thapa

Department of Obstetrics and Gynaecology

Tribhuvan University Teaching Hospital

Kathmandu, Nepal.

Email : thapamira@hotmail.com

Phone: 9851001133 


\section{CASE REPORT}

A 25-year-old second gravida, presented to emergency department with the complaint of persistent lower abdominal pain for five days. The pain was severe, continuous and decreased with antispasmodics. Her last menstrual period was 14 weeks ago. No history of vaginal bleeding was present. She was sexually active without contraception. No history of irregular menstruation, abortions, sexually transmitted diseases, pelvic inflammatory disease, ovulatory drugs or assisted reproduction was present. She had one living child, delivered by normal vaginal delivery four years back.

On examination, her general condition was satisfactory with the pulse rate of 100 beats per minute and blood pressure of $120 / 80 \mathrm{~mm} \mathrm{Hg}$. Abdominal examination was not much significant with absent guarding, rigidity and tenderness. Vaginal speculum examination revealed normal cervix with no bleeding and on bimanual examination uterus was normal size with tender fornices and an ill defined mass of approximately $4 \times 4 \mathrm{~cm}$ was felt in the right fornix. Cervical movement was tender.

Her hemoglobin was $12.3 \mathrm{~g} / \mathrm{dl}$. Urine for pregnancy test was positive. Ultrasonography revealed an anteverted empty uterus of normal size and echotexture, with normal endometrium. A sac like structure was seen in right adnexal region along with the embryonic pole, but without cardiac activity (Figure 1). There was significant amount of collection in the peritoneal cavity and on diagnostic peritoneal aspiration, blood was recovered. A provisional diagnosis of ruptured ectopic pregnancy was made.

Emergency laparotomy revealed enlarged and ruptured right ovary revealing material that looked like products of conception along with hemoperitoneum containing 300 grams of blood clot and $700 \mathrm{ml}$ of blood. The right fallopian tube with fimbria was congested. The left ovary, fallopian tube with fimbria and uterus were normal.

Right sided oophorectomy was performed as the ovary had ruptured. The postoperative period was uneventful. Histopathological examination showed ovarian tissue with chorionic villi lined by cytotrophoblast and syncytiotrophoblast trapped in the blood clot.

\section{DISCUSSION}

Ovarian pregnancies are classified as primary and secondary. Primary occurs if the ovum is fertilized while still within the follicle and this phenomenon is postulated to be a consequence of ovulatory dysfunction. In the secondary type, there is a tubal abortion with secondary implantation of the embryo in the ovary. ${ }^{1}$ Primary ovarian pregnancy is a rare entity; the reported incidence being 1 in $25,000-40,000$ pregnancies, $0.5-3 \%$ of extrauterine pregnancies. ${ }^{2,3}$

Although increase in incidence of ovarian pregnancy is due to wider use of IUCD, ovulatory drugs and assisted reproductive techniques, no such history were present in this patient.

The diagnosis of an ovarian ectopic pregnancy is seldom made before surgery. At the time of surgery the diagnosis was made as a hemorrhagic mass seen attached to one of the ovaries in the presence of normal looking fallopian tubes (Figure 2). ${ }^{4}$

The recent advances in ${ }_{\mathbf{B}} \mathrm{HCG}$ determination and transvaginal ultrasound have aided the diagnosis. A serum вhCG level of $1500 \mathrm{mlU} / \mathrm{mL}$ in the absence of an intrauterine sac is highly suggestive of an ectopic pregnancy. Ultrasound, especially transvaginal scanning has proven to be an invaluable tool in the diagnosis of this condition. Even then, it can be mistaken for a hemorrhagic corpus luteum or ovarian cyst. ${ }^{5}$ We too performed a urinary вhCG test which was positive along with USG revealing sac like structure in the right adnexal region with significant intra-abdominal collection suggestive of ectopic pregnancy but not that of ovarian.

Spiegelberg suggested four criteria to distinguish a primary ovarian pregnancy from a disturbed pregnancy, which has secondarily involved the ovary. ${ }^{6}$ They are:

1. The fallopian tube with its fimbria should be intact and separate from the ovary.

2. The gestational sac should occupy the normal position of the ovary.

3. The gestational sac should be connected to the uterus by the ovarian ligament.

4. Ovarian tissue must be present in the specimen attached to the gestational sac.

Laparotomy findings and histopathological report of the resected ovary were consistent with the Spiegelberg criteria, suggesting it to be an ovarian ectopic pregnancy.

With early detection of uncomplicated cases; laparoscopic surgery is the main method of treatment for ovarian ectopic pregnancies. Early detection of an ovarian pregnancy prior to rupture of the gestational sac and onset of active bleeding permits laparoscopic surgery and removal of the ectopic pregnancy without excessive removal of healthy ovarian tissue. ${ }^{4,7,8}$ But this was not possible in our case due to hemoperitoneum and lack of laparoscopic surgery facility.

\section{\begin{tabular}{l|l|l|l|l|l|l|} 
JNMA & VIL 49 & Na. 1 & ISSUE & 177 & JAN-MAR, 201
\end{tabular}}


There is a place for medical treatment (Methotrexate) in selected patients with ectopic pregnancies either tubal or ovarian. It prevents possible surgical complications such as intraoperative hemorrhage, oophorectomy and pelvic adhesions. ${ }^{4}$

Review reports reveal that about $91.0 \%$ of ovarian pregnancies get terminated in the first trimester, $5.3 \%$ in the second trimester and $3.7 \%$ in the third trimester. Only one case of ovarian pregnancy which had progressed to full-term delivery has been reported. ${ }^{9}$ Usually

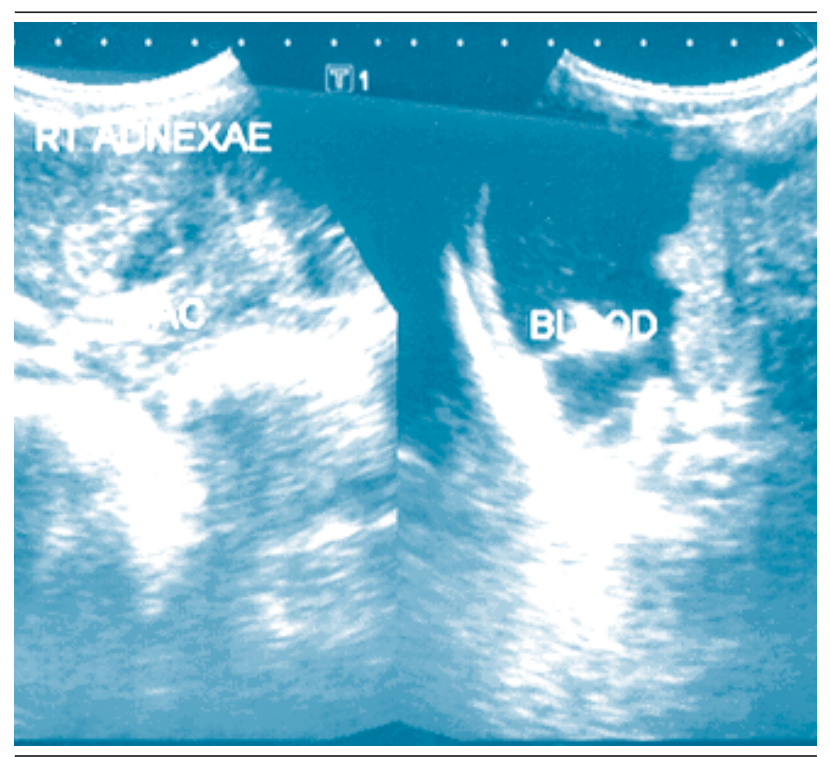

Figure 1. USG picture showing sac in right adnexa and hemoperitoneum

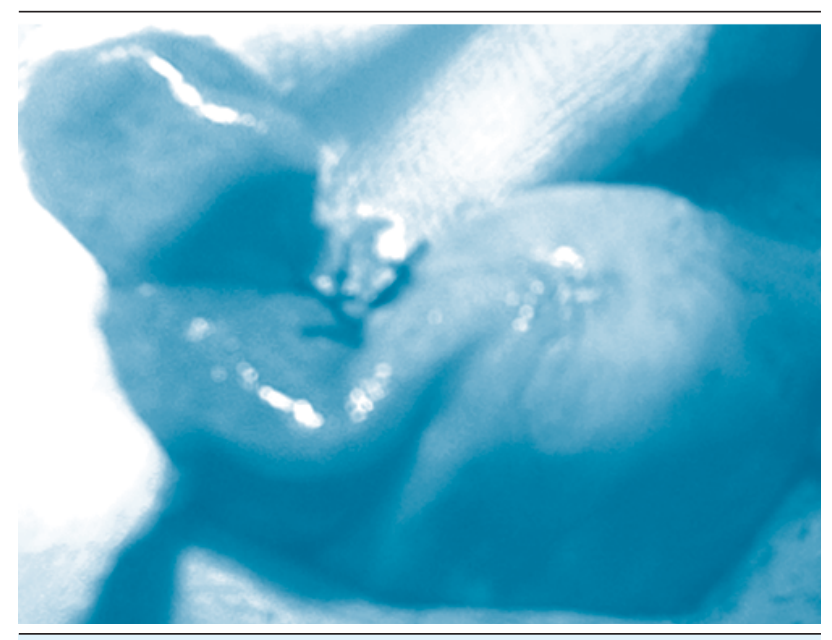

Figure $2 \mathrm{~b}$. Intraoperative picture after right sided oophorectomy performed postoperative period will be uneventful. In these patients fertility is conserved, recurrence is rare unlike tubal pregnancy and the future pregnancies are usually intrauterine. $^{10}$

Following hCG concentrations postoperatively (until negative) is important since remaining chorionic villi may be able to grow postoperatively. If this does occur, adjuvant therapy with methotrexate to destroy any remaining pregnancy tissue is generally effective. ${ }^{11}$ Our patient was asked to have regular follow up examination.

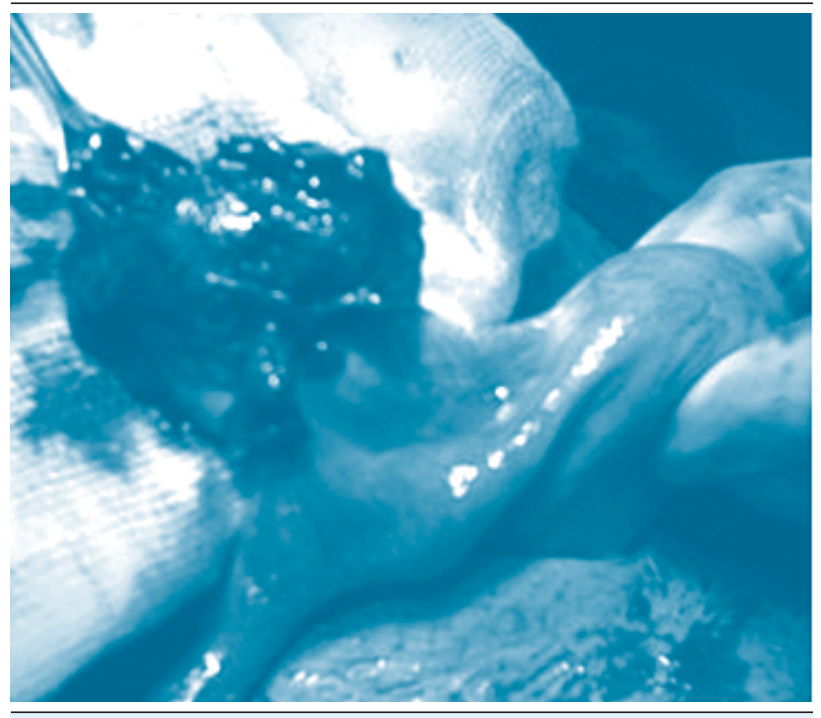

Figure 2a. Intraoperative picture showing normal uterus, fallopian tube and ruptured right ovary with product of conception like material and blood clot

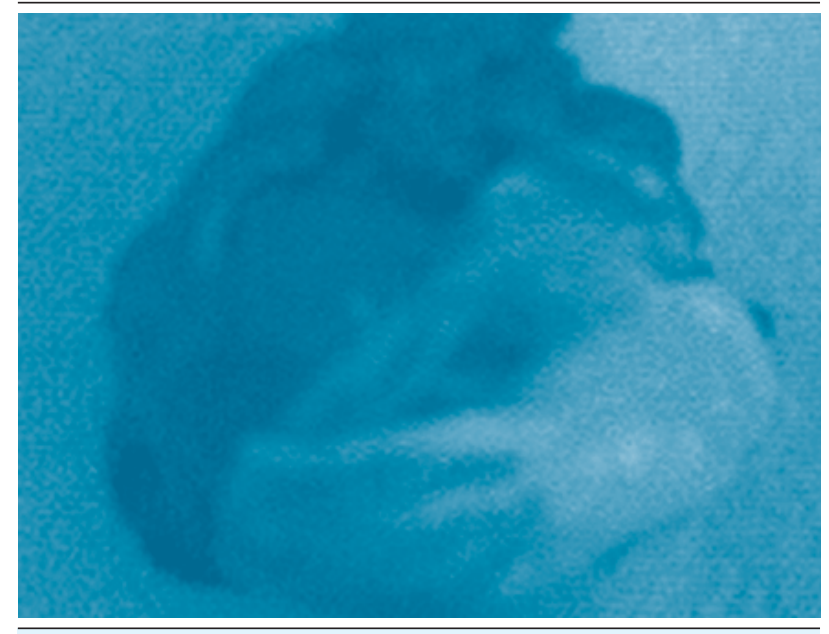

Figure 2c. Resected specimen sent for histopathological examination 


\section{REFERENCES}

1. Das S, Kalyani R, Lakshmi V, Harendra Kumar ML. Ovarian pregnancy. Indian J Pathol Microbiol. 2008;51:37-8.

2. López E, Zarama F, Builtron R. Embarazo ectópico abdominal con producto vivo. Presentación de un caso. Ginec Obst Mex. 2001;69:151-4.

3. Bontis J, Grimbizis G, Tariatzis B, et al. Ovarian pregnancy after ovulation induction / intrauterine insemination: pathophysiological aspects and diagnostic problems. Human Reproduction. 1997;12:376-8.

4. Nicholas F, Schwartz L. Primary ovarian pregnancy successfully treated with methotrexate. Am J of Obstet Gynecol. 1992;167(5):1307-8 .

5. Russel JB, Cutler LR. Transvaginal ultrasonographic detection of primary ovarian pregnancy with laparoscopic removal. Fertil Steril. 1989;51:1055.
6. Spiegelberg. Cauistik der Ovarial schwangerschaft. Arch E Gynak. 1878;13:73-6.

7. Nadarajah S, Sim LN, Loh SF. Laparoscopic Management of an Ovarian Pregnancy. Singapore Med J. 2002;43(2):95-6.

8. Tjalma WA, Tjalma AA. Ovarian tumor: ovarian pregnancy. Diagnosis and management. Clin Exp Obstet Gynecol. 2003;30(23):122-4.

9. William PC, Machar TC, Icneft JR. Term ovarian pregnancy with delivery of female infant. Am J Obstet Gynecol. 1982;142:1589-91.

10. Gray CL, Ruffolo EH. Ovarian pregnancy associated with intrauterine contraceptive devices. Obstet Gynecol. 1978;10:1324 .

11. Farghaly M, Saeed M. Primary ovarian pregnancy. ASJOG. 2005;2:297-9. 